\title{
Suchmaschinen reflektiert nutzen
}

im Landkreis Nordsachsen

\author{
Lernende Maschinen erfordern denkende Nutzer - ein Überblick
}

\author{
von Dr. Björn Brückerhoff
}

In der Mediengeschichte hat die Entwicklung neuer Medien stets zu einem „Zugewinn der Materialerschließung und Kanalisierung von Datenströmen“ (Kümmel/Scholz/Schumacher 2004: 8) geführt, zugleich aber „,neue Formen von Unübersichtlichkeit“ (ebd.) erzeugt. Das führte jeweils zur Einrichtung von Meta-Orientierungsangeboten, die die Nutzer über die zur Nutzung vorliegenden Medieninhalte orientieren und damit die Nutzbarkeit der Medien sicherstellen sollten. Zu nennen sind etwa Inhaltsverzeichnisse, Bibliographien, Trailer oder Programmzeitschriften (Brückerhoff 2019: 62-69): „Mehr Medien erforderten bislang also noch mehr Medien, um nutzbar zu bleiben“ (Brückerhoff 2019: 17), ein „Informationsparadoxon“ (Kuhlen 1999: 173) entsteht. Universalsuchmaschinen und ihre Weiterentwicklungen, die digitalen Assistenten, führen dagegen potenziell zu einem Paradigmenwechsel: Mit der ständigen Ausweitung ihrer Orientierungs- und Meta-Orientierungsmöglichkeiten (zu den Orientierungsbegriffen: Brückerhoff 2019 55-68) kann die Entstehung weiterer Meta-Orientierungs- oder gar Meta-Meta-Orientierungsangebote obsolet werden. „Die Eigenschaften des Mediums, über das orientiert werden soll, bedingen die notwendigen Eigenschaften der Meta-Orientierungsangebote, die Meta-Orientierungsleistungen darüber erbringen sollen." (Brückerhoff 2019: 21). Entsprechend erfordert das „Konvergenzmedium“ (Neuberger 2011: 40) Internet Meta-Orientierungsangebote, die konvergierenden Medieninhalten gerecht werden. Neben den Meta-Orientierungsleistungen über Medieninhalte liefern hochentwickelte Suchmaschinen zudem seit Jahren Orientierungsleistungen für ihre Nutzer, geben also selbst eigene Inhalte aus, statt nur via Hyperlink-Listen auf Inhalte Dritter zu verweisen.

Dabei stellt sich die Frage, wie die mittels Universalsuchmaschinen und digitalen Assistenten erbrachten (Meta-)Orientierungsleistungen zu bewerten sind und auf welche Weise sie zur Orientierung ihrer Nutzer beitragen können - Fragen, die in Zeiten populärer Verschwörungserzählungen, Fake News und postfaktischer Tendenzen in der Gesellschaft durchaus brisant sind (Brückerhoff 2018). Was müssen Suchmaschinennutzer also wissen, um Suchmaschinen reflektiert nutzen zu können?

\section{Übersichtliche Zustände: Die Anfänge}

Früher war die Frage nach den erforderlichen Kompetenzen vergleichsweise einfach zu beantworten. Wer zu Beginn der Neunzigerjahre mit den Vorläufern heutiger Suchmaschinen, Programmen wie Archie oder Veronica, arbeitete, war Expertin oder Experte. Suchanfragen hatten stark strukturiert zu sein, die Programme erstellten Listen und leiteten ihre Nutzer zu Dateien weiter. Die Auswahl der Dateien seitens der Suchmaschine erfolgte gemäß der Eingaben der Nutzer, eine Bewertung ihrer Relevanz musste ausschließlich durch die Nutzer erfolgen. Als Nachfolger dieser Information Retrieval-Systeme entstanden mit dem Aufkommen des World Wide Web ab 1993 die ersten Suchmaschinen, zum Beispiel der World Wide Web Wanderer und - etwas später - Lycos (1994) und AltaVista (1995). Die ersten Web-Suchmaschinen arbeiteten in der Regel textstatistisch. So wurden als Suchergebnisse Verweise auf Websites ausgegeben, die beispielsweise bestimmte Schlagwörter besonders häufig aufwiesen. Die Ergebnisse waren oft unzureichend, eine Manipulation war vergleichsweise einfach. Diese Suchmaschinen richteten sich vornehmlich an Laien, die im noch jungen World Wide Web selbst über kaum nennenswerte Nutzungserfahrungen verfügen konnten. Wer mehrere Begriffe oder gar eine Frage in das Suchfeld eingab, erhielt häufig unpassende oder gar keine Ergebnisse. Um Erfolg zu haben, war die Verwendung einer Suchsyntax obligatorisch, die sich jedoch in der Praxis häufig auf die Anwendung boolescher Operatoren (UND/ODER) beschränkt haben dürfte. Auch hier mussten die Nutzer die Ergebnisse selbst beurteilen und für sich optimale Entscheidungen treffen, auch wenn die Suchmaschinen bei gelungener Eingabe bereits eine gewichtete Reihenfolge ausgaben.

Die Frage, wie sich die genutzte Suchmaschine finanziert, war für die Nutzer innen und ihre Nutzungserfahrungen noch nachrangig. Die Finanzierung verlief zumeist über Bannerwerbung. Im weiteren Verlauf der Entwicklung richtete sich das Interesse der Anbieter - grob zusammengefasst - darauf, die Bedienung zu vereinfachen, die subjektive Relevanz der ausgegebenen Meta-Orientierungsleistungen zu erhöhen, ihre Manipulierbarkeit zu reduzieren (Brückerhoff 2019: 160) und damit auch als Werbeplattform attraktiver zu werden.

\section{Entwicklung zu Universalsuchmaschinen und digitalen Assistenten}

Mit der Ausweitung der über das Internet verfügbaren Inhalte und ihrer Ausdifferenzierung sind Spezialsuchmaschinen entstanden, die beispielsweise die Suche nach bestimmten Dateiformaten (Audio-, Bilder-, Videosuche), Themenfeldern oder wissenschaftlichen Dokumenten (Brückerhoff 2019: 162-167) ermöglichten. Zudem führten die Digitalisierungsprojekte von Suchmaschinenanbietern, insbesondere von Google, zur digitalen Erschließung vormals nicht digitaler Medieninhalte, etwa von Büchern, Landkarten, Fotos, Gemälden, Filmen oder Musikstücken (Brückerhoff 2019: 192-193). Auch integrierten Anbieter wie Google und Bing (Microsoft) Inhalte, ,die ursprünglich keine Medieninhalte waren, etwa Satellitenbilder, oder Inhalte, die neu für die Nutzung im Orientierungsbereich Internet erstellt worden sind, beispielsweise digital fotografierte Straßen- und Landschaftsansichten, Fotografien von Gebäudeinnenräumen oder Museumsbeständen“ (Brückerhoff 2019: 84).

Die Trennung von allgemein ausgerichteten Suchmaschinen und Spezialsuchmaschinen löst sich inzwischen auf. Ihre Suchergebnisse werden in die Ergebnisdarstellung der allgemein ausgerichteten Suchmaschinen integriert, die dadurch zu Universalsuchmaschinen werden. Sie haben den Anspruch, neben den über das Internet erreichbaren Datenbeständen selbstständig alle Daten zu erfassen, die digital erreichbar sind, also auch außerhalb des World Wide Web vorliegen.

Führende Anbieter des Suchmaschinenmarktes, insbesondere Google, fördern durch die Schaffung eigener Dienstleistungsangebote die Ent-

Herausgeber

Redaktion

Dr. Benjamin Bigl

Medienpädagogisches Zentrumplus

Dr. Benjamin Bigl

Puschkinstraße 3

04860 Torgau

\section{Prof Dr. Denise Sommer}

Befunde aus Journalismus, Medien und Gesellschaft Literatur- und Medientipps geben Anregungen zu Vertiefung und Thematisierung in der Bildungsarbeit. TransferPlus wird auf Qucosa, dem sächsischen Open- 
stehung von Daten im Alltag der Nutzer, die dann erfasst und ausgewertet werden können. Die ohnehin zunehmende Digitalisierung des Alltags begünstigt diesen Prozess und erzeugt für die Nutzer innen neue Orientierungsbedürfnisse, die von Suchmaschinen befriedigt werden können.

Google erfasst beispielsweise detaillierte Nutzungsdaten mittels der eigenen Suchmaschinen, über das eigene Werbeanzeigen-Netzwerk, die zahlreichen Google-Apps, das Betriebssystem Android und über Google-Smartphones sowie Smart Home-Geräte. Die Datensammlung umfasst potenziell die Auswertung der Internetnutzung (stationär und mobil), den Zugriff auf Bilder und Videos, auf Gesprächsthemen, Termine, Aufenthaltsorte, Reiseplanungen, persönliche Kontakte, Bewegungsprofile und Nutzungsgewohnheiten. Google kennt den Klang der Stimmen seiner Nutzer, kann potenziell geplante und tatsächliche Kaufhandlungen erfassen, auf die Häufigkeit und die Art sportlicher Aktivitäten sowie auf über Apps erfasste Gesundheitsdaten zugreifen. Über Smart Home-Anwendungen ist ferner die Datenerfassung zu Hause möglich, zum Beispiel die Erstellung von Live-Videoaufnahmen aus der Wohnung der Nutzer innen, die Erhebung von Daten zur Anwesenheit zu Hause und die Erstellung von Bewegungsprofilen in der eigenen Wohnung. Sogar Daten zur Zusammensetzung der Raumluft (über Rauchmeldesysteme) sind denkbar - die Liste lässt sich fortsetzen. Universalsuchmaschinen und digitale Assistenten können aus diesen Daten zum Beispiel Rückschlüsse auf die Wünsche und Bedürfnisse, die sexuelle Orientierung, die physische und psychische Gesundheit, die politischen Meinungen, die finanziellen Möglichkeiten, die religiösen Überzeugungen oder die räumliche Flexibilität der Nutzer_innen ziehen. Auch der Blick auf die Daten im Zeitverlauf ist attraktiv: Abweichungen von gewohnten Abläufen im Alltag können erkannt, der Gesundheitszustand kann protokolliert und prognostiziert werden (bis hin zum Versuch der Prognose der Lebenserwartung) - die Analysemöglichkeiten scheinen unbegrenzt. Neben der Nutzung dieser Daten zur Erbringung von Orientierungs- und Meta-Orientierungsleistungen sind sie auch für die Finanzierung über Werbung ideal, da sie nicht nur potenziell subjektiv höchstrelevante Ergebnisse versprechen, sondern auch die Platzierung von Werbebotschaften mit nie gekannter Präzision gestatten. Darüber hinaus müssen Suchmaschinen-Anbieter ihre Funktionsweisen und die ständigen Modifikationen ihres Angebots intransparent halten, um ,plausible und subjektiv relevante Ergebnisse generieren zu können, die das Vertrauen der Nutzer begründen“ (Brückerhoff 2019: 213). So sichern sich Suchmaschinen-Anbieter gegenüber ihren Wettbewerbern oder anderen externen Interessengruppen ab (Brückerhoff 2019: 214-216) und versuchen, Beeinflussungsversuche zu minimieren. Das ist natürlich eine Gratwanderung: „Die unklare Arbeits- und Funktionsweise durch die notwendige Geheimhaltung der Algorithmen und die technische Selektion auf Basis einer extensiven und zudem intransparent erfolgenden Datensammlung stehen [zugleich] dem Vertrauen der Nutzer entgegen“" (Brückerhoff 2019: 213). Was bedeutet diese Situation für die reflektierte Nutzung?

\section{Schlussfolgerungen}

Den Suchmaschinen-Anbietern dürfte klar sein, dass die exzessive Datensammlung und die notwendige Intransparenz des Vorgehens das dringend notwendige Vertrauen der Nutzer gefährden kann. Zugleich ist die Datensammlung sowohl für den Betrieb hochentwickelter Such- maschinen und die - teils selbstständig erfolgende - Weiterentwicklung der integrierten Künstlichen Intelligenz unabdingbar. Natürlich gibt es auch heute Suchmaschinen, die auf eine derart umfangreiche Datensammlung verzichten - und damit sogar werben, zum Beispiel Startpage oder DuckDuckGo. Die Marktführer haben jedoch die nächste Stufe bereits gezündet: Mittels künstlicher Intelligenz unternehmen Suchmaschinen und digitale Assistenten auf Basis der erhobenen Daten den Versuch, die Orientierungsbedürfnisse ihrer Nutzer innen zu antizipieren, bevor diese artikuliert werden. Abgesehen davon, dass dieses Unterfangen auch misslingen kann, besteht die Gefahr der Kultivierung einer übermäßigen Bequemlichkeit der Nutzer_innen, die durch Spracheingabe und Sprachausgabe potenziell noch verstärkt wird. Dadurch treten möglicherweise Quellenangaben in den Hintergrund oder verschwinden sogar ganz. Das erschwert oder verhindert zum Beispiel die intersubjektive Nachprüfbarkeit der Ergebnisse. Gewöhnungseffekte könnten folgen - und die Herkunft der Daten könnte im Zeitverlauf für die Nutzer_innen immer weniger relevant sein. Geschäftsmodelle, die Datenerhebungen sowie Intransparenz voraussetzen und gleichzeitig das Vertrauen der Nutzer erfordern, hat beispielsweise Zuboff (2019) ausführlich analysiert („Surveillance Capitalism“). Ob diese Geschäftsmodelle funktionieren, liegt an den Nutzern. Kurz: Suchmaschinen entwickeln sich zwar zu vorausschauenden digitalen Assistenten, die sehr bequem zu nutzen sind - denken sollten die Nutzer_innen allerdings weiterhin selbst.

\section{Quellen}

Brückerhoff, B. (2018): Fake News als Symptom postfaktischer Politik. In: Brückerhoff, B. (Hrsg.): Neue Gegenwart. Magazin für Medienjournalismus. Kommunikation in den Zeiten des digitalen Kulturwandels. Ausgabe 60/2018. http://www.neuegegenwart.de/ ausgabe60/postfaktisch.htm

Brückerhoff, B. (2019): Orientierung durch Suchmaschinen. Ein dynamisch-transaktiona gedachtes Modell. Köln: Herbert von Halem.

Kümmel, A.; Scholz, L.; Schumacher, E. (Hrsg.) (2004): Einführung in die Geschichte der Medien. Paderborn: Wilhelm Fink.

Kuhlen, R. (1999): Die Konsequenzen von Informationsassistenten. Was bedeutet informationelle Autonomie oder wie kann Vertrauen in elektronische Dienste in offenen Informationsmärkten gesichert werden? Frankfurt/Main: Suhrkamp.

Neuberger, C. (2011): Soziale Netzwerke im Internet. Kommunikationswissenschaftliche Einordnung und Forschungsüberblick. In: Neuberger, C.; Gehrau, V. (Hrsg.): StudiVZ. Diffusion, Nutzung und Wirkung eines sozialen Netzwerks im Internet. Wiesbaden: VS/ Springer Fachmedien, S. 33-96.

Zuboff, S. (2019): The Age of Surveillance Capitalism. The Fight for a Human Future at the New Frontier of Power. London: Profile.

Autor der Ausgabe

Dr. Björn Brückerhoff (Jg. 1979) ist Journalist, Autor, Dozent und Berater. Schwerpunkt Medien und digitale Transformation (www.brueckerhoff.de). Für das von inm seit 1998 herausgegebene und gestaltete Online-Medienmagazin Neue Gegenwart (www.neuegegenwart.de) hat er u. a. den Grimme Online Award erhalten. Kontakt: buero@brueckerhoff.de.

\section{Empfohlene Zitation des Beitrages}

Brückerhoff, B. (2020): Suchmaschinen reflektiert nutzen. Lernende Maschinen erfordern denkende Nutzer - ein Überblick. In: B. Big (Hrsg.). Transfer Pus - Aktuelle Beiträge zur Medienbildung. Issue 02/2020. dol: $10.25366 / 2020.13$

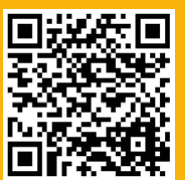

Die Politik des Suchens (Bundeszentrale für politische Bildung)
Orientierung durch Suchmaschinen. Ein dynamisch-transaktional gedachtes Modell (Herbert von Halem Verlag)

Liferatur

Rushkoff, D. (2019): Team Human. New York: Norton.

Zuboff, S. (2019): The Age of Surveillance Capitalism. The Fight for a Human Future at the New Frontier of Power. London: Profile. 